\title{
Prevention of Pressure Ulcer in Intensive Care Units
}

\author{
Fatemeh Ahmadian, ${ }^{1}$ Davood Hekmatpou, ${ }^{1,}$ Maryam Eghbali, ${ }^{2}$ and Shadi Farsaei ${ }^{3}$ \\ ${ }^{1}$ Nursing and Midwifery Faculty, Arak University of Medical Sciences, Arak, IR Iran \\ ${ }^{2}$ Isfahan Cardiovascular Research Center, Department of Intensive Care Nursing, Nursing and Midwifery Faculty, Isfahan University of Medical Sciences, Isfahan, IR Iran \\ ${ }^{3}$ Pharmacology Faculty, Isfahan University of Medical Sciences, Isfahan, IR Iran \\ "Corresponding author: Davood Hekmatpou, Nursing and Midwifery Faculty, Arak University of Medical Sciences, Arak, IR Iran. Tel: +98-9181616539, Fax: +98-634173528, E-mail: \\ dr_hekmat@arakmu.ac.ir
}

Received 2016 April 06; Accepted 2016 April 11.

\section{Abstract}

Background: One of the most common problems in intensive care units (ICUs) is pressure ulcer or bed sore and its prevention is a priority in nursing cares.

Objectives: The current study aimed to evaluate the effect of henna (Lawsonia inermis) on prevention of pressure ulcers in patients in ICU.

Patients and Methods: The current randomized clinical trial was conducted on 80 patients with the inclusion criteria hospitalized in the ICU of Al-Zahra hospital, Isfahan, Iran in 2015. Patients were randomly allocated into two groups of control (40 patients) and intervention (40 patients) by drawing method. For the intervention group, along with the standard prevention cares for pressure ulcers, once a $15 \mathrm{~cm}$ thick layer of henna was applied on patients' sacrum. Data was gathered using demographic data questionnaire, Braden scale to predict pressure ulcer risk and the table to control daily record of skin temperature before the intervention for a week. Data were analyzed using SPSS ver. 21 and Chi-square, Greenhouse Geisser, Pearson correlation coefficient and independent t-test were used in the study.

Results: The mean age of patients in the intervention and control groups was $51.35 \pm 17$ and $52.94 \pm 19.61 y e a r s$, respectively. The means of standard deviation score of the Braden scale for all the participants on the first, fourth and seventh day of the study were $12.27 \pm 2.85,14.23 \pm 3.21$ and $15.73 \pm 3.82$, respectively, which had significant differences with each other $(\mathrm{P}<0.0001)$; suggesting that the risk of pressure ulcers occurrence was higher on the first day than the seventh day. There was a reverse relationship between reduction of the Braden scale score and increase in local warmth of sacrum $(r=0.558)$ in both groups. The mean and standard deviation of local warmth of sacrum during the seven days of study were $37.84 \pm 0.52$ and $38.06 \pm 0.67$ in the intervention and control groups, but their difference was insignificant $(\mathrm{P}<0.14)$. The mean and standard deviation of local warmth of forehead during the seven days of study were $37.09 \pm 0.41$ and $37.03 \pm 0.44$ in the intervention and control groups but their difference was insignificant $(\mathrm{P}<0.5)$. There was a statistically significant difference between the mean local warmth of sacrum and forehead in the intervention group during the seven days of study $(\mathrm{P}=0.0001)$. At the end of the study, one patient in the intervention group (2.7\% male) and six patients in the control group (14.29\% male, $2.85 \%$ female) had developed pressure ulcers; this difference was significant $(\mathrm{P}=0.001)$.

Conclusions: Considering the effect of henna in reducing the local warmth, redness, and inflammation of sacrum in patients in intensive care units, application of henna to prevent pressure ulcers for patients at risk of developing pressure ulcers is recommended.

Keywords: Intensive Care Unit, Henna, Pressure Ulcer, The Braden Scale, Iran, Lawsonia inermis

\section{Background}

Wounds and their treatments are as old as human beings. One of the most common health care problems is pressure ulcers or sore bed (1). A pressure ulcer is a local damage to skin or its underneath tissues that usually occurs around bony prominences due to pressure or a combination of pressure and friction of sliding forces (2-5).

The occurrence and prevalence of pressure ulcers are mostly the indicators of nursing care quality (6). There are different studies on the prevalence of stage 1 pressure ulcers in various hospitals and wards. Comparing the to- tal number of hospitalizations, the prevalence and occurrence rate of pressure ulcers is mostly done among patients in intensive care units (ICUs) (2). Various studies reported the prevalence of pressure ulcers in intensive care units between $10 \%$ and $41 \%$ (7). In Iran, in a study conducted by Ali Akbari Sari et al. (2014) the prevalence of pressure ulcers in ICUs was 26.7\% (8).

These ulcers cause pain and discomfort great harm to the quality of life and they are also costly (9). Pressure ulcers are the third costly disorder after cancer and cardiovascular diseases $(10,11)$. According to the statistics in 2012, about 1.6 million patients annually develop pres- 
sure ulcers in ICUs and it costs USD11-17.2 billion (2). Patients, families, medical health centers and society are significantly affected by physical, financial and social consequences of pressure ulcers and patients with pressure ulcers inevitably experience pain, malformation, disability and dependence on others (12). According to the statics, each pressure ulcer, along with increased pain, adds four days to hospitalization time in America and also increases the risk of nosocomial infections by $25 \%$ (10). The complications of pressure ulcers increase the rate of mortality among patients by 55\% (13). Each pressure ulcer imposes $50 \%$ more workload to nurses (14). The undesirable consequences and high treatment costs make it a priority to prevent pressure ulcers (10).

Preventing pressure ulcers is the priority of nursing cares (15). Gunningberg et al. in a prospective study reported that almost $90 \%$ of pressure ulcers could be prevented by accurate predictions and appropriate nursing interventions (9). According to the European pressure ulcer advisory panel (EPUAP) and national pressure ulcer advisory panel in 2009, pressure ulcers preventive measures include: evaluating the risk of pressure ulcers, evaluating the skin and skin care, appropriate diet, changing the position and using supportive surfaces to reduce pressure (3). Nowadays using traditional medicine and herbal remedies are among the methods to prevent pressure ulcers.

The world health organization (WHO) (2003) reported that $80 \%$ of the population of all countries cannot afford pharmaceutical drugs and refer to traditional medicines, based on herbs, to support their primary health needs (16, 17). Henna (Lawsonia inermis) is a medicinal herb that different studies mentioned the therapeutic effect of its leaf on preventing and treating diabetic foot ulcers (18), treating hand-foot syndrome caused by drug reactions (19) and treating acute and chronic stomach ulcers in rats (16).

\section{Objectives}

The current study aimed to evaluate the effect of henna on prevention of pressure ulcers considering the history of henna consumption and also its various pharmacologic effects including antibacterial, antifungal, antiviral, antimicrobial, anti-parasitic (20-22), analgesic, antiinflammatory, antipyretic (19, 20, 23), immune stimulus, anti-skin diseases, antioxidant, anti-tuberculosis, (20) and lack of scientific researches regarding its effect on preventing pressure ulcers.

\section{Patients and Methods}

This two-group clinical trial was conducted under registration number IRCT2015070323035N1 in 2015 in ICUs of
Al-Zahra hospital, Isfahan, Iran. The inclusion criteria were :age range 18 - 75 years old, willingness to participate in the study, not having any pressure ulcers at the time of admission, probable continuous hospitalization at ICU, being admitted to ICU in the last 24 hours and not being hospitalized at any other wards before admission to ICU, not having diabetes, having a systolic blood pressure of $100 \mathrm{mmHg}$ or more, not being addicted to narcotics, not having anemia (hemoglobin level of less than $12 \mathrm{~g} / \mathrm{dL}$ in males and $10 \mathrm{gr} / \mathrm{dL}$ in females) or any other blood diseases and not having a fever $(\mathrm{T} \leq 37.5)$. The exclusion criteria were unwillingness to continue with the study or death of the patient.

After being approved and receiving permission from the ethics committee of the university and also taking written informed consent form from patients' families, 80 patients with the inclusion criteria were selected through targeted sampling and randomly allocated into two groups of control and intervention using drawing method. Then daily standards to prevent pressure ulcers were applied to all the patients by nurses. Along with the standard measures, to prevent pressure ulcers in the intervention group, first henna extract was provided (by mixing one gram of powdered leaves of henna with $10 \mathrm{~mL}$ of distilled water) and applied on the inner part of the forearm and if the patient showed no allergic reaction to henna, the prepared henna (combination of fifty grams of powdered henna leaves and $500 \mathrm{~mL}$ of distilled water) was applied on the sacrum with a thick $15 \mathrm{~cm}$ layer. The applied henna was left on the skin for 30 minutes and then it was rinsed with warm water and the skin was dried. No particular measure was applied to the control group.

Data were gathered through demographic questionnaire at the time of admission by the researcher, Braden scale to predict pressure ulcer risk was completed on the first, fourth and seventh day of the study by the researcher (the Braden scale is a valid tool to evaluate the risk of pressure ulcers in the guideline of wounds, ostomy and continence nurses society). It includes six dimensions of sensory perception, humidity, mobility, activity, diet and friction force. This scale is scored from 6 (highest risk) to 23 (lowest risk) (2). According to this scale a score of nine or lower indicates extremely high risk, 10 -12 is high risk, 13-14 is moderate risk, 15 - 18 is low risk and a score of more than 18 indicates no risk (11) and the table to control daily record of sacrum and forehead temperature was completed by a trained nurse using a Microlife infrared thermometer, made in Switzerland, every day at 9 a.m. Any increase in the sacrum temperature of the intervention group compared to the control group was considered a local inflammation and stage 1 pressure ulcer. The stage of the ulcer was also determined according to the definition of European pressure ulcer advisory panel (3). Studying the par- 
ticipants continued for one week, in case of not developing any pressure ulcer (of course in both groups occurrence of permanent and indelible redness by pressuring the finger was also considered as the indicator of occurrence of stage 1 pressure ulcer (3). Data were analyzed using SPSS ver. 21 and Chi-square, Greenhouse, Pearson correlation and independent $\mathrm{t}$-test were used in the study.

\section{Results}

In the current study, $78.4 \%$ of the subjects in the intervention group were male and $21.6 \%$ were female and in the control group $62.9 \%$ were male and $37.1 \%$ were female. Most of the patients in the intervention (51.4\%) and the control (65.7\%) groups had endotracheal tube. The medical diagnosis for most of the patients of the intervention group (45.9\%) was brain damage and for the control group (37.1\%) was trauma. None of the studied participants had a history of deep vein thrombosis (DVT). In the intervention group the highest frequency of previous surgeries belonged to urinary tract and reproductive system surgery (16.2\%) and in the control group belonged to cardiovascular system surgery (17.1\%). Most of the patients in the intervention (54.1\%) and control (54.3\%) groups had underlying diseases; $27 \%$ of the intervention group and $34.3 \%$ of the control group subjects had cardiovascular problems; $73 \%$ of the intervention group and $77.1 \%$ of the control group subjects had no history of narcotic consumption and from those who had a history of substance abuse, most of them (21.6\% in the intervention group and $14.3 \%$ in the control group) were cigarette smokers. Data analysis showed that both groups had no significant statistical differences regarding their demographic characteristics and other variables before the intervention $(\mathrm{P}>0.05)$ (Table 1).

Results showed that the mean score of the Braden scale on the first, fourth and seventh day of the study for both the control and the intervention groups were $12.27 \pm 2.85$, $14.23 \pm 3.21$ and $15.73 \pm 3.82$, respectively. Statistical tests showed a significant difference between the mean scores at three different times $(\mathrm{P}<0.0001)$. The mean score of the Braden scale for the intervention and the control groups on the first day were $12.75 \pm 3.07$ and $11.77 \pm 2.55$, respectively; on the fourth day were $14.89 \pm 3.42$ and $13.54 \pm 2.86$, respectively; and on the seventh day were $16.24 \pm 3.78$ and $15.20 \pm 3.84$, respectively. Statistical tests showed no significant interaction between the mean scores of the two groups at three different times $(\mathrm{P}<0.755)$, indicating that the mean scores were not significantly different in the two groups (Figure 1).

Regarding the risk of pressure ulcers using the Braden scale on the first, fourth and seventh day of the study, statistical tests showed that on the first day $75.6 \%$ of the interven-
Table 1. Comparing Demographic Characteristics of the Two Intervention and Control Groups and Henna Impact on the Prevention of Pressure Ulcers in Intensive Care Units

\begin{tabular}{lccc}
\hline $\begin{array}{l}\text { Demographic } \\
\text { Characteristics }\end{array}$ & Intervention & Control & Test Results \\
& Mean \pm SD & Mean \pm SD & P \\
\hline Age & $51.35 \pm 17$ & $52.94 \pm 19.61$ & 0.7 \\
\hline BMI & $23.85 \pm 3.19$ & $24.43 \pm 3.72$ & 0.4 \\
\hline Consciousness level & $10.59 \pm 3.58$ & $9.40 \pm 3.83$ & 0.17 \\
\hline Serum albumin & $3.31 \pm 0.63$ & $3.16 \pm 0.60$ & 0.3 \\
\hline $\begin{array}{l}\text { Probable duration of } \\
\text { hospitalization }\end{array}$ & $7.51 \pm 1.70$ & $7.71 \pm 1.93$ & 0.6 \\
\hline $\begin{array}{l}\text { Systole blood pressure } \\
\text { Diastole blood } \\
\text { pressure }\end{array}$ & $127.84 \pm 15.8$ & $130.67 \pm 15.08$ & 0.4 \\
\hline a BMI, body mass index & $77.93 \pm 8.8$ & $78.62 \pm 8.7$ & 0.7 \\
\hline
\end{tabular}

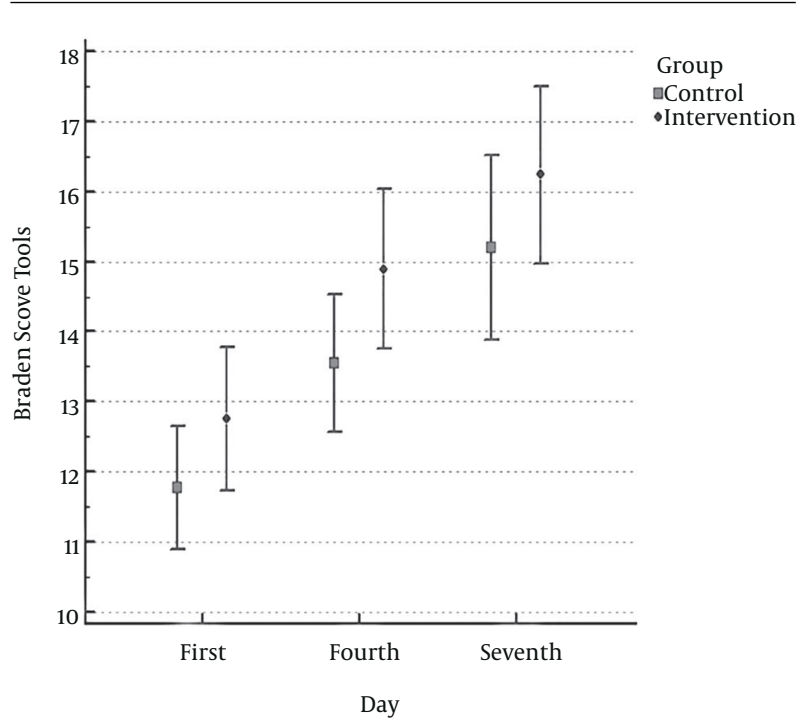

Figure 1. The Analysis of Variance Among the Subjects Based on the Braden Score on Three Occasions (First, Fourth and Seventh Study) in Both Intervention and Control Groups

tion group and $85.7 \%$ of the control group subjects, on the fourth day $45.9 \%$ of the intervention group and $68.6 \%$ of the control group subjects and on the seventh day $35.1 \%$ of the intervention group $51.4 \%$ of the control group subjects had a moderate to high risk of developing pressure ulcers (score of 6 -14). Based on the Fisher exact test, there was no significant difference between the two groups regarding the risk of developing pressure ulcers $(\mathrm{P}>0.5)$. Also results showed that most of the participants had a score of 10 - 12 (high risk) on the first day and a score of more than 18 (no risk) on the seventh day of the study (Table 2). 
Table 2. The Risk of Pressure Ulcer Using the Braden Scale During the First, Fourth and Seventh Days in the Intervention and Control Groups ${ }^{\mathrm{a}}$

\begin{tabular}{|c|c|c|c|c|c|c|}
\hline \multirow[t]{2}{*}{ Day Risk of Pressure Ulcers } & \multicolumn{3}{|c|}{ Intervention } & \multicolumn{3}{|c|}{ Control } \\
\hline & First & Fourth & Seventh & First & Fourth & Seventh \\
\hline Moderate to high risk (score 6 - 14 ) & $28(75.6)$ & $17(45.9)$ & $13(35.1)$ & $30(85.7)$ & $24(68.6)$ & $18(51.4)$ \\
\hline Low risk (score 15 - 18) & 13.5 & $12(32.4)$ & $9(24.3)$ & $3(8.6)$ & $7(20)$ & $6(17.1)$ \\
\hline No risk (score 18 - 23) & 10.8 & $8(21.6)$ & $15(40.5)$ & $2(5.7)$ & $4(11.4)$ & $11(31.4)$ \\
\hline
\end{tabular}

${ }^{\mathrm{a}}$ Values are expressed as No. (\%).

Results showed that in the control and intervention groups during all the seven days of study, there was a relationship between decrease in the score of the Braden scale and increase in local warmth of sacrum $(r=-0.409, r=$ 0.558 , respectively).

The mean local warmth of the sacrum during the seven days of the study in the intervention and the control groups were $37.84 \pm 0.52$ and $38.06 \pm 0.67$, respectively. From the beginning of the study (first day) till its end (seventh day) the mean local warmth of sacrum in the intervention group was lower than that of the control group but their difference was not statistically significant $(\mathrm{P}=0.14)$. According to independent t-test, only at the beginning of the study the difference between the mean local warmth of sacrum of both groups was significant $(\mathrm{P}=0.001)$ (Figure 2).

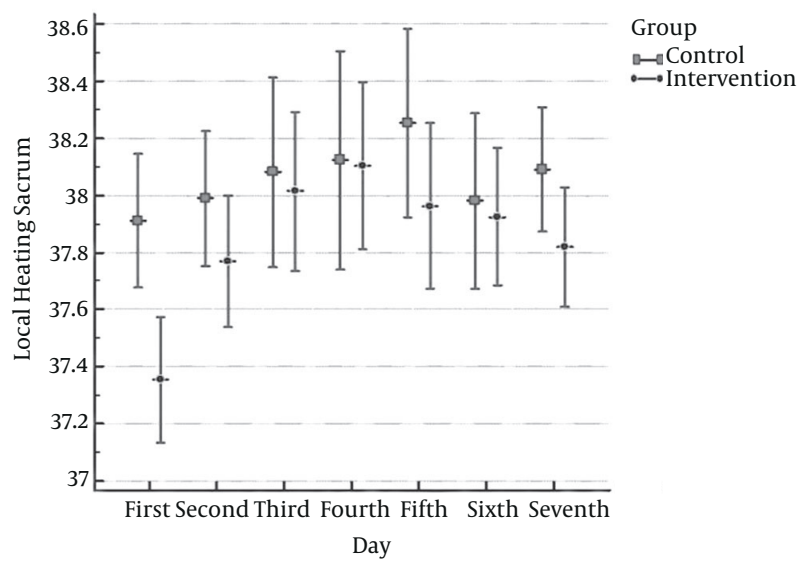

Figure 2. The Analysis of Variance Between the Mean Temperature of the Sacrum on Weekdays in Both Intervention and Control Groups

The mean warmth of forehead during seven days of the study was $37.09 \pm 0.41$ in the intervention group and 37.03 \pm 0.44 in the control group. There was no significant difference between the groups regarding the mean warmth of their forehead $(\mathrm{P}=0.5)$ (Figure 3).

Regarding the relationship between the mean of body

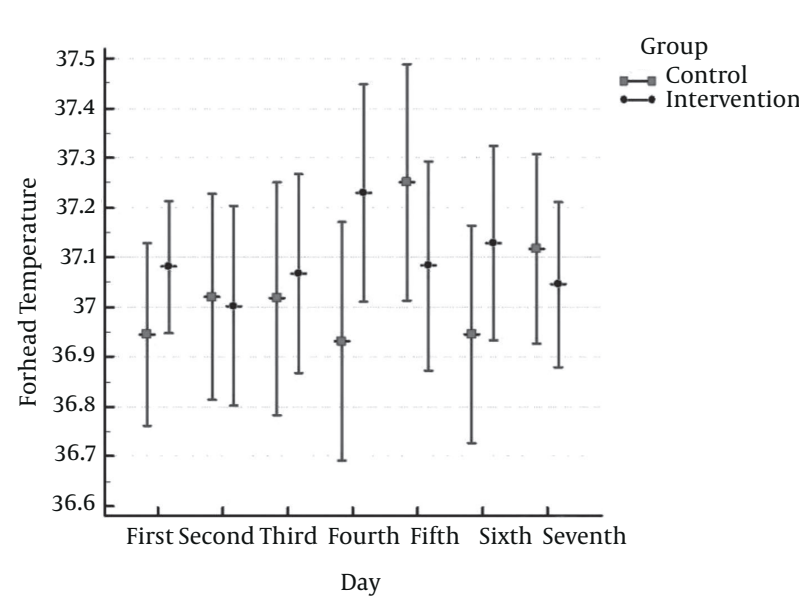

Figure 3. The Analysis of Variance Between the Mean Temperature of the Forehead on Weekdays in Both Intervention and Control Groups

temperature (forehead) with local warmth of sacrum in both groups after the study, independent t-test showed that the total mean score of sacrum of the intervention group was lower than that of the control group, but their difference was insignificant $(\mathrm{P}=0.14)$. The total mean score of forehead temperature in the intervention group was higher than that of the control group but the difference was not significant $(P=0.5)$. Also the results of the Pearson correlation coefficient showed a significant relationship between the increase in the mean local warmth of sacrum and increase in forehead temperature during the seven days of the study; although there was a stronger association in the intervention group $(\mathrm{r}=0.677)$.

Daily observations showed that one patient in the intervention group and six patients in the control group had redness in the sacral region. The comparison of the two rates showed that the difference between the groups regarding redness in the sacral region on the sixth and seventh days of the study was significant $(\mathrm{P}=0.01)$. Results showed that one patient in the intervention group (one male, $2.70 \%$ ) and six patients in the control group (five males, $14.29 \%$ and one female, $2.85 \%$ ) developed pressure 
ulcers at the end of the study and this difference was significant $(\mathrm{P}=0.001)$.

\section{Discussion}

Eighty patients with the inclusion criteria participated in the current study and were allocated into two equal groups of intervention and control. During the study eight patients (six males and two females) were excluded from the study. Six patients died during the study and the other two did not cooperate. The study continued with 72 patients; 35 in the control group and 37 in the intervention group. Results showed that both groups were similar regarding gender, age, intubation condition, medical diagnosis, history of DVT, history of surgery, history of underlying diseases, history of substance abuse, body mass index (BMI), level of consciousness, serum albumin level, probable duration of hospitalization, blood pressure and the risk of developing pressure; ulcers and statistical tests found no significant differences between them. Therefore, it could be said that both groups were similar.

Regarding the mean score of risk of developing pressure ulcers using the Braden scale for all patients, results showed that the mean score of the Braden scale on the first, fourth and seventh day of the study both for the control and the intervention groups was 12.27, 14.23 and 15.73, respectively and the difference between the mean scores at three different times was significant; indicating that all the patients in the two groups had a lower score in the Braden scale on the first day of the study than the seventh day, which indicates higher risk of developing pressure ulcers on the first day of admission. This increase in score could be explained by the recovery process of the patients after seven days of hospitalization. Therefore on the first days of admission, due to less activity and decreased level of consciousness, the patients are at higher risk of developing pressure ulcers. Jiang et al. showed that $11.79 \%$ of the patients with the Braden scale score of less than 17 had a high risk of developing pressure ulcers (4). Also in the study by Eberlein-Gonska et al. decrease in the score of Braden scale was one of the risk factors of developing pressure ulcers during hospitalization (5). Results of these studies were similar to the results of the present study.

Results of the current study showed no significant difference between the mean score of the Braden scale at three different times (first, fourth and seventh day of the study) in the intervention and the control groups. It means that the mean score of both groups were similar, which indicates that both groups were similar regarding the risk of developing pressure ulcers based on the Braden scale. Soozani et al. showed that the mean scores of wound assessment in patients who developed pressure ulcers was
$13.8 \pm 3.6$ and in those who did not was $20.2 \pm 3.5$; the Baden scale and independent t-test showed that the difference between the groups was significant (11). Regarding the risk of developing pressure ulcers using the Braden scale, the results of the present study were similar to those of other studies. Jiang et al. showed that $11.79 \%$ had a high risk of developing pressure ulcers and their score of the Braden scale was less than 17; this group needed more attention to prevent developing pressure ulcers. Also $88.21 \%$ of the patients with a score of 17 or more in the Braden scale had no risk of developing pressure ulcers (4). In the study by Lupianez-Perez et al., one of the inclusion criteria was diagnosis of the risk of developing skin ulcers by the Braden scale conducted by the nurse; a score of 12 or less was considered high risk of developing pressure ulcers and a score of 13 - 16 was considered moderate risk (6).

In the control and intervention groups, there was a relationship between decrease in the score of Braden scale and decrease in local warmth of sacrum, indicating that the lower the score of Braden scale, the higher the risk of developing pressure ulcers. Ulker Efteli et al. showed that low score of the Braden scale indicated low level of activity and hence high risk of developing pressure ulcers (9). The study by Amirifar et al. (10) revealed that the lower the score of the Braden scale, the higher the risk of developing pressure ulcers. As it could be observed, the results of these studies are similar to those of the current study.

Although in the present study the local warmth of sacrum, from the first to the seventh day of the study, was lower in the intervention group than the control group, the difference between the groups was only significant on the first day. Since in the intervention group the prepared henna was applied on sacrum and rinsed with warm water after 30 minutes and then patients' skin were dried, they had lower local warmth of sacrum; but the control group did not receive any special measures. According to EPUAP (2009), to diagnose pressure ulcers skin observation should evaluate the warmth of location and edema or stiffness, especially in patients with dark skin. Warmth, edema and stiffness are indicators of developing pressure ulcers since the signs of pressure ulcers or redness could not always be observed in patients with dark skin (3). Therefore, it could be implied that increase in the local warmth of sacrum could indicate development of pressure ulcer and based on the results of the present study, since the mean local warmth of sacrum was lower in the intervention group, it could be concluded that applying henna on sacrum might have been effective in lowering the local warmth of sacrum and somehow preventing increased warmth, which is an indicator of developing pressure ulcers.

Although throughout the study the mean warmth of 
sacrum was lower in the intervention group than the control group, their difference was not statistically significant. In other words, the difference between the mean of forehead temperature, which indicates the body temperature, was not significant in the groups. Therefore, it could be concluded that the two groups were comparable. The study by Mogarehi and Sanaiey (24) mentioned that the mean body temperature of the patients with pressure ulcers was $37.2^{\circ} \mathrm{C}$ and that of the patients without pressure ulcers was $37^{\circ} \mathrm{C}$, the difference was not significant. Braden and Bergstrom reported that the body temperature in patients with pressure ulcer was higher.

According to the results of the present study, in both groups the warmth of sacrum was higher than that of forehead temperature but the difference between sacrum's temperature and forehead temperature in the intervention group was less than those of the control group; indicating that the warmth of sacrum in the intervention group increased less than that of the control group, which indicates lower risk of developing pressure ulcers among the patients of intervention group. The results also showed that the mean temperature of sacrum and forehead had a significant relationship during the study; the relationship between local warmth of sacrum and forehead temperature was direct. Akbari Sari et al. (8) mentioned that fever by changing patient's metabolism on one hand and sweating on the other hand would make the patient prone to developing pressure ulcers (25). This is similar to the results of the present study.

In the present study only one patient in the intervention group developed redness in sacrum on the third, fourth and fifth day of the study and on the sixth and seventh day of the study, the redness disappeared, but in the control group redness occurred in six patients. The difference was significant. According to the EPUAP (2009) the definition of stage 1 pressure ulcer includes healthy skin with a pale red or a different color region that usually occurs around bony prominences and could be more painful, smoother, softer, warmer or colder than adjacent regions. Redness means that body is not able to release from the previous pressures and needs rest before being pressured again (3). Considering the above mentioned points it could be said that one patient in the intervention group and six patients in the control group developed pressure ulcers and the difference was significant in this regard. Therefore, the applied intervention was effective and prevented redness in sacrum and eventually pressure ulcer, especially stage 1. Yucel et al. evaluated the effect of henna on handfoot syndrome caused by capecitabine and henna, due to its anti-inflammatory, analgesic and antipyretic effects, it could have a good therapeutic effect on reduction of this syndrome without the need to reduce drug dosage (19).
According to the results one patient in the intervention group developed stage 1 pressure ulcer, which the redness in the sacrum region was resolved after 72 hours, and in the control group five patients developed stage 1 pressure ulcer and one patient developed stage 2 ulcer. EberleinGonska et al. revealed that the lower stages of pressure ulcers (stage 1 or 2 ) are more common than their higher grades (stage 3 or 4 ) in such a way that $41 \%$ of the patients had stage 1 (784 patients out of 1914 ) and $48 \%$ had stage 2 (915 patients) pressure ulcers (5). In the study by Mistiaen et al., conducted for three years from 2006 to 2008 , nurses who visited patients at home reported all the cases of pressure ulcer at sacrum region and out of the 2772 patients, 1517, 820, 288 and 147 patients had stages 1, 2, 3 and 4 ulcers, respectively (26). In the study by Soozani et al. (11) conducted in Iran on 72 patients, 53 (73.6\%) had stage 2 and $16(22.2 \%)$ had stage 1 pressure ulcers. In most of the studies, stage 1 and 2 ulcers were more common, similar to the present study. Considering that most of the ulcers have low grades and their treatments are costly, preventing these ulcers is a high priority. The present study showed that one patient in the intervention group and six patients in the control group developed pressure ulcers. Therefore, it could be said that applying henna on sacrum could have been effective in preventing pressure ulcers and could be suggested as a clinical preventive method.

\subsection{Conclusions}

Considering the above mentioned points about the effects of henna in preventing pressure ulcers and also the results of the present study, it could be suggested that henna is effective in preventing pressure ulcers, but due to the limited time of the study and small sample size it is recommended to conduct a long-term study on a larger sample size.

\section{References}

1. Azimian J, Pourkhaleghi E, Ansari M. Assessing the topical high pressure oxygen therapy in healing of bedsore. JQUMS. 2010.

2. Potter P, Perry A, Stockert P, Hall A. Fundamentals of nursing. eighth ed. $2 . ; 2013$.

3. EPUAP. Prevention and treatment of pressure ulcers: quick reference guide. Washington DC 2009. Available from: WWW.npuap.org.

4. Jiang Q, Li X, Qu X, Liu Y, Zhang L, Su C, et al. The incidence, risk factors and characteristics of pressure ulcers in hospitalized patients in China. Int J Clin Exp Pathol. 2014;7(5):2587-94. [PubMed: 24966973].

5. Eberlein-Gonska M, Petzold T, Helass G, Albrecht DM, Schmitt J. The incidence and determinants of decubitus ulcers in hospital care: an analysis of routine quality management data at a university hospital. Dtsch Arztebl Int. 2013;110(33-34):550-6. doi: 10.3238/arztebl.2013.0550. [PubMed: 24069079].

6. Lupianez-Perez I, Morilla-Herrera JC, Ginel-Mendoza L, Martin-Santos FJ, Navarro-Moya FJ, Sepulveda-Guerra RP, et al. Effectiveness of olive 
oil for the prevention of pressure ulcers caused in immobilized patients within the scope of primary health care: study protocol for a randomized controlled trial. Trials. 2013;14:348. doi: 10.1186/1745-621514-348. [PubMed: 24152576].

7. Cooper KL. Evidence-based prevention of pressure ulcers in the intensive care unit. Crit Care Nurse. 2013;33(6):57-66. doi: 10.4037/ccn2013985. [PubMed: 24293556].

8. Akbari Sari A, Doshmanghir L, Neghahban Z, Ghiasipour M, Beheshtizavareh Z. Rate of Pressure Ulcers in Intensive Units and General Wards of Iranian Hospitals and Methods for Their Detection. Iran J Public Health. 2014;43(6):787-92. [PubMed: 26110149].

9. Ulker Efteli E, Yapucu Gunes U. A prospective, descriptive study of risk factors related to pressure ulcer development among patients in intensive care units. Ostomy Wound Manage. 2013;59(7):22-7. [PubMed: 23846003].

10. Amirifar S, Reza Masouleh S, Pourshikhian M, Monfared A, Kazemnejad Leili E. Predictive value of Braden Scale in pressure ulcer occurrence in hospitalized patients. J Holist Nurs. 2013;23(2):8-15

11. Soozani A, Khosravi A, Pourheydari M, MONTAZERI A. Using Braden and Waterlow scales to predict pressure ulcer: A comparative study. Knowledge Health. 2011;5(4).

12. Bansal C, Scott R, Stewart D, Cockerell CJ. Decubitus ulcers: a review of the literature. Int J Dermatol. 2005;44(10):805-10. doi: 10.1111/j.13654632.2005.02636.x. [PubMed: 16207179].

13. Berwick DM. protecting 5 million lives from harm Available from: http://www.ihi.org/engage/initiatives/completed/ 5MillionLivesCampaign/Pages/default.aspx.

14. Shahin ES, Dassen T, Halfens RJ. Incidence, prevention and treatment of pressure ulcers in intensive care patients: a longitudinal study. Int J Nurs Stud. 2009;46(4):413-21. doi: 10.1016/j.ijnurstu.2008.02.011. [PubMed: 18394626].

15. Aydin AK, Karadag A. Assessment of nurses' knowledge and practice in prevention and management of deep tissue injury and stage I pressure ulcer. J Wound Ostomy Continence Nurs. 2010;37(5):487-94. doi: 10.1097/WON.ob013e3181edecob. [PubMed: 20736857].

16. Goswami M, Kulshreshtha M, Rao C, Yadav S, Yadav S. Anti-ulcer poten- tial of Lawsonia inermis L. Leaves against gastric ulcers in rats. IJPS. 2011;1(2):69.

17. Nesa L, Munira S, Mollika S, Islam M, Choin H, Chouduri AU, et al. Evaluation of analgesic, anti-inflammatory and CNS depressant activities of methanolic extract of Lawsonia inermis barks in mice. Avicenna J Phytomed. 2014;4(4):287-96. [PubMed: 25068143].

18. Mutluoglu M, Uzun G. Can henna prevent ulceration in diabetic feet at high risk?. Exp Diabetes Res. 2009;2009:107496. doi: 10.1155/2009/107496. [PubMed: 20016805].

19. Yucel I, Guzin G. Topical henna for capecitabine induced hand-foot syndrome. Invest New Drugs. 2008;26(2):189-92. doi: 10.1007/s10637007-9082-3. [PubMed: 17885735].

20. Chaudhary G, Goyal S, Poonia P. Lawsonia inermis Linnaeus: a phytopharmacological review. ijpsdr. 2010;2(2):91-8.

21. Babu PD, Subhasree RS. Antimicrobial activities of Lawsonia inermisa review. Acad J Plant Sci. 2009;2(4):231-2.

22. Arulpriya $P$, Lalitha $P$. The wound healing potential of aerial roots of Rhaphidophora aurea (Linden ex Andre) climbed over Lawsonia inermis. Asian J Pharm Clin Res. 2013;6(1):132-5.

23. Dasgupta T, Rao AR, Yadava PK. Modulatory effect of henna leaf (Lawsonia inermis) on drug metabolising phase I and phase II enzymes, antioxidant enzymes, lipid peroxidation and chemically induced skin and forestomach papillomagenesis in mice. Mol Cell Biochem. 2003;245(1-2):11-22. [PubMed: 12708740].

24. Mogarehi M, Sanaiey Z. Pressure sore incidence and risk factors in patients admitted in medical, surgical and orthopedic wards in affiliated hospitals of Shiraz University of medical sciences. Iran Journal of Nursing. 2003;16(34):8-13.

25. Beheshti Zavare Z, Arab M, Rashidian A, Golestan B. Factors affecting pressure ulcer in the ICU units of Tehran University of Medical Sciences teaching hospitals. Sjsph. 2010;8(3):81-92.

26. Mistiaen P, Ament A, Francke AL, Achterberg W, Halfens R, Huizinga J, et al. An economic appraisal of the Australian Medical Sheepskin for the prevention of sacral pressure ulcers from a nursing home perspective. BMC Health Serv Res. 2010;10:226. doi: 10.1186/1472-6963-10226. [PubMed: 20687915]. 